\title{
Quantitative characterization of molecular-stream separation
}

\author{
Sven Kochmann and Sergey N. Krylov* \\ Department of Chemistry and Centre for Research on Biomolecular Interactions, York University, Toronto, Ontario \\ M3J 1P3, Canada
}

\begin{abstract}
Molecular-stream separation (MSS), e.g. by free flow electrophoresis or continuous annular chromatography, has great potential for applications that require continuous downstream separation such continuous flow synthesis. Despite its potential, MSS still needs to be greatly advanced, which requires currently lacking tools for quantitative characterization of streams in MSS. We developed and introduce

here an analytical toolbox for this task. The first tool is a method to convolute 3D raw MSS data into a 2D "angulagram" via signal integration over the whole separation zone using a polar coordinate system. The second tool is three quantitative parameters characterizing stream width, linearity, and deflection, which are determined from an angulagram. The third tool is the analysis of the three parameters in relation to physicochemical characteristics of MSS which reveals deficiencies and guides improvements in MSS devices and methods. Examples of toolbox application to validation of previously published MSS data are provided.
\end{abstract}

\section{INTRODUCTION}

Separation of molecules plays a pivotal role in many molecular analyses and in purification of molecular components of complex mixtures. Molecular separation - both analytical and preparative - can be achieved by chromatography and electrophoresis, which have many similarities despite differences in separation mechanisms. The movement of molecules in both separation approaches can be characterised by two velocity vectors: $\vec{v}_{\text {flow }}$, the velocity of a molecule-dragging flow (pressure-driven or electroosmotic), and $\vec{v}_{\text {disp }}$, the velocity of displacement of molecules under the influence of a discriminative separating force ("phasedistributional" or electrostatic); ${ }^{1,2}$ the magnitudes of these vectors are $v_{\text {flow }}$ and $v_{\text {disp }}$, respectively. In the context of this manuscript, we consider $\vec{v}_{\text {flow }}$ and $\vec{v}_{\text {disp }}$ each to be unidirectional, which is the most common case. If $v_{\text {flow }}=0$ or $\vec{v}_{\text {flow }} \| \vec{v}_{\text {disp }}$ then only 1D plug separation is possible as in common column chromatography and capillary electrophoresis (Figure 1a). ${ }^{3,4}$ If $v_{\text {flow }} \neq 0$ and $\vec{v}_{\text {flow }} \nVdash \vec{v}_{\text {disp }}$, then 2D molecular-stream separation (MSS, our term) can be realized as in continuous annular chromatography (CAC) and free flow electrophoresis (FFE) (Figure 1b).

MSS has great potential for applications that require continuous downstream separation. For instance, MSS has been recently suggested as a potentially powerful complement to continuous-flow synthesis; such a combination promises steady-state continuous-flow synthesis with seamless downstream purification. ${ }^{8-10}$ Despite its potential, MSS still needs to be greatly advanced in both instrumentation and methodology to achieve a level suitable for its practical application in downstream separations. This work was motivated by our insight that achieving this level of advancement will be impossible without an analytical toolbox for quantitative characterization of streams in MSS.
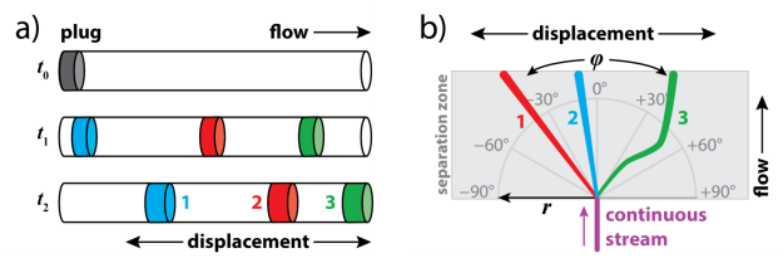

Figure 1. Plug (a) vs stream (b) separation. Panel (a): A short plug of a multicomponent mixture is injected and its components are separated while travelling through the column or capillary. Panel (b): A continuous stream of a multicomponent mixture is introduced into a separation zone and individual streams of components are separated from each other. Polar coordinates are a natural way to describe geometry of streams that fan out from the origin of coordinates.

MSS analysis is analytically and technically challenging due to the high dimensionality of its data sets. MSS developers have no convenient and customized method for this task. Up to now, developers circumvent the lack of an appropriate analytical toolbox by extracting single graphs from cross sections, usually towards the end of the separation zone. ${ }^{11-14}$ If available, these cross section graphs are complemented by an (further unevaluated) image of the separation zone for a general overview. Evaluation of such cross section graphs seems convenient, but turns a blind eye to the large parts of the remaining separation zone. However, for full quantitative characterization of streams in MSS the evaluation of the whole separation zone is needed.

In this work, we address the lack of an appropriate analytical toolbox. We propose and introduce a set of tools for the quantitative characterization of streams in MSS, which are simple to use and serve as a starting point for further advancement of MSS and its analysis. 


\section{RESULTS AND DISCUSSION}

Stream quality and characteristics. A separation zone in MSS (Figure 1b) is a thin channel with a flow axis (along $\vec{v}_{\text {flow }}$ ) and a displacement axis (along $\vec{v}_{\text {disp }}$ ) being non-parallel to each other. Ideally, each of $\vec{v}_{\text {flow }}$ and $\vec{v}_{\text {disp }}$ is uniform within the entire channel and the molecular streams fan out as straight narrow lines (e.g. Streams 1 and 2 in Figure 1b). In reality, $\vec{v}_{\text {flow }}$ and $\vec{v}_{\text {disp }}$ are non-uniform in the flow-displacement plane, which leads to stream non-linearity (e.g. Stream 3 ), and $\vec{v}_{\text {disp }}$ is not uniform along the height of the channel (perpendicular to the flow-displacement plane) which leads to stream broadening (Streams 1-3). Stream quality depends on all of these velocities and non-uniformities.

Stream quality in MSS can be fully described by stream deflection, stream width, and stream linearity. Low stream quality, i.e. non-linear and broad streams with insufficient and/or incorrect stream deflection, is indicative of specific imperfections in fabrication and operation of the MSS device (discussed below). These imperfections are important to find and address rationally. Quantitative characterization of streams in MSS must, thus, involve finding quantitative parameters that unambiguously characterize stream deflection, stream width, and stream linearity.

We need to mathematically define such parameters in a way that allows their determination from raw MSS data (lightabsorbance or fluorescence images in a wide spectral range). Raw MSS data are 3D (optical signal as function of two coordinates), which makes defining such parameters very difficult. Any practical approach to defining and determining the three parameters must first reduce the dimensionality of raw MSS data to 2D without losing necessary information. The first analytical tool to develop for the proposed toolbox was, thus, a method for reducing the dimensionality of raw MSS data without losing necessary information.

Reducing dimensionality of raw MSS data. To reduce the dimensionality of raw MSS data without losing necessary information, we propose to convolute the 3D raw MSS data to their 2D complement via integration of the signal along the streams over the whole separation zone. Given uniform flow fields, streams fan out from the same inlet point and progress into the separation zone at different angles to $\vec{v}_{\text {flow }}(e . g$. Streams 1 and 2 in Figure 1b). Naturally, a polar coordinate system with angle $\varphi$, radian $r$, and the origin at the inlet can be used to describe MSS separation (Figure 1b and SI 1, we use $\mathrm{SI}$ in combination with the section number to refer to specific parts of the Supporting Information).

Raw MSS data can be convoluted by signal integration over $r$ :

$$
S(\varphi)=\int_{r=0}^{r_{\max }(\varphi)} s(\varphi, r) d r
$$

where $s(\varphi, r)$ is the signal intensity in point $(\varphi, r)$ and $r_{\max }(\varphi)$ is the maximum radian within the separation zone for each value of $\varphi$ (Figure 2a, b). We refer to $S(\varphi)$ as "angulagram" due to the dependence of $S$ solely on the angle $\varphi$.

Analysis of angulagrams. Constructing an angulagram does not require any information about the scale or dimensions of the respective MSS separation zone. This property has two advantages. First, an angulagram can be built directly from a digital image of the signal in the separation zone (SI 2); the only information required is pixel coordinates of the inlet (i.e. a)
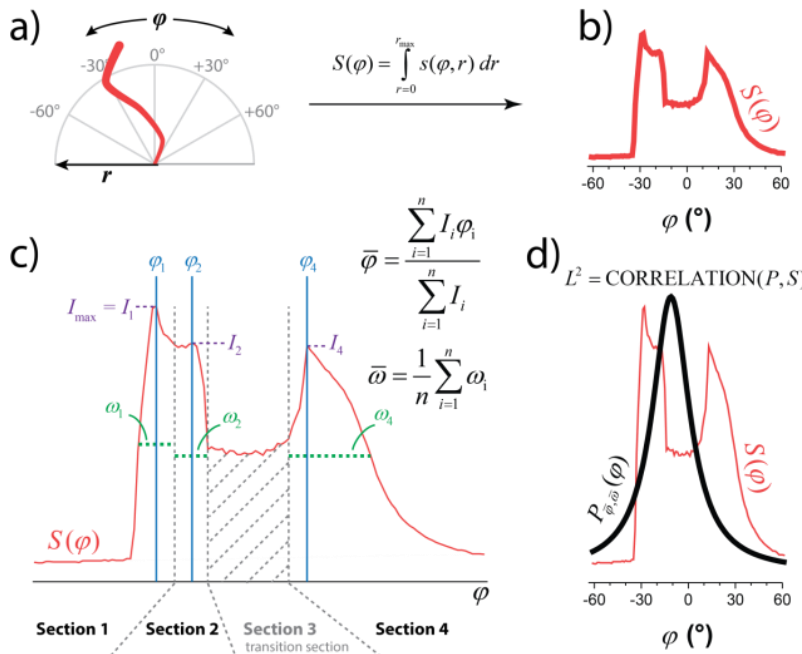

$\varphi\left(^{\circ}\right)$

d)

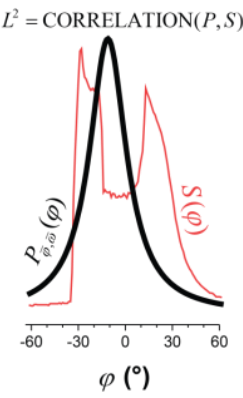

Figure 2. Constructing and dissecting an angulagram for quantitative characterization of an MSS stream. Schematic raw MSS data of a tottering stream (a) is convoluted into an angulagram (b) which reveals a multi-feature peak with shoulders and valleys. Dissection of this peak (c) allows extracting stream deflection $\bar{\varphi}$ and stream width $\bar{\omega}$, which are calculated with the formulas shown in the top right corner. Linearity is calculated (d) by determining the correlation between the measured signal $S(\varphi)$ and an ideal peak shape $P(\varphi)$ constructed with $\bar{\varphi}$ and $\bar{\omega}$. See SI 5 for more information.

the origin of coordinates) on the image. Therefore, an angulagram can be constructed retrospectively from previously published images (SI 3). Secondly, angulagrams obtained from separations of differently scaled devices can be directly compared to each other; normalization of intensity is not required for comparison since the crucial information for assessing the stream quality are functions of $\varphi$ and use the intensity only as relative measure, which is described in detail in the following subsections. Note that the stream quality parameters themselves might need normalization (e.g. to account for different electric fields in different FFE devices).

With uniform velocity fields and minimal broadening, a MSS stream results in a symmetrical Lorentzian peak in an angulagram (Figure 3a). Stream disturbance due to a nonuniform velocity field results in a multi-feature peak containing shoulders or splitting in various degrees (Figure 3bd). Such a peak must be dissected and its features must be analysed as exemplified in Figure 2c and SI 5. Looking forward, an angulagram contains all necessary information for defining the three parameters characterizing stream deflection, stream width, and stream linearity (Figure 2c,d); it also contains more of interpretable and useful information (SI 4). Now that the 3D raw MSS data have been convoluted into a $2 \mathrm{D}$ angulagram, we can focus on developing the second tool for the proposed toolbox: quantitative parameters characterizing stream deflection, stream width, and stream linearity determined from an angulagram. They must be defined from information contained in an angulagram.

Peak intensity and area in angulagrams. The peak intensities and areas in an angulagram depend on various things, e.g. the number of signal points along $r$ or the actual signal intensities, i.e. $s(\varphi, r)$ (Eq. 1). These peak intensities and areas can provide information about the represented streams if the nature of signal (e.g. fluorescence) and its parameters (e.g. 

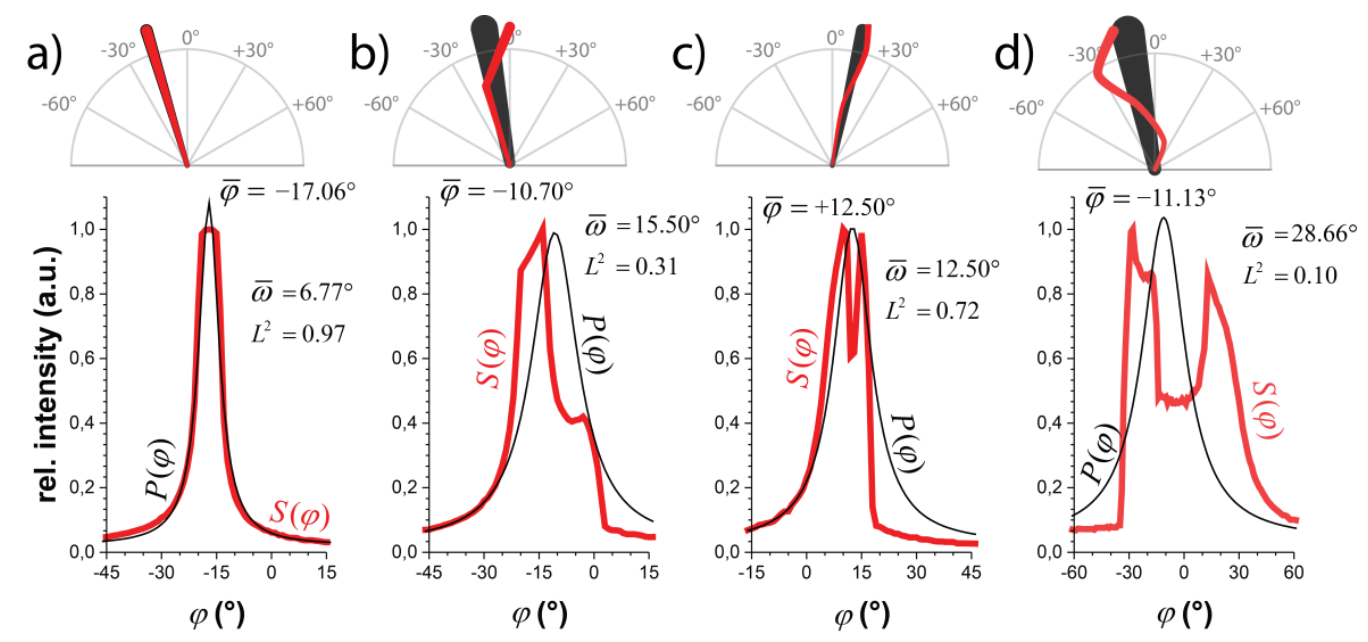

Figure 3. Examples of different stream shapes (schemes at the top) resulting in different angulagrams (plots at the bottom) and different quantitative parameters for stream deflection, stream width, and stream linearity for an ideal stream (a), bending stream (b), wiggling stream (c), and tottering stream $(\mathbf{d})$.

molar extinction coefficient, quantum yield, etc.) is known. For instance, the intensity can be a measure for the length of the stream or the total amount of substance in the separation zone. For absolute measures, a reference standard is required in most cases. If this information is not needed, the intensities can be normalized by normalizing $r_{\max }(\varphi)$ during the integration step (Eq. 1) to avoid making small-intensity peaks looking 'worse' or 'weaker'. The usefulness or necessity for such normalization depends on the actual evaluation case; in this manuscript, we refrain from this procedure to keep our descriptions and discussions as general as possible.

Stream deflection. To characterize stream deflection, we suggest an average peak position $\bar{\varphi}$ defined by weighting the individual maxima on the peak with their intensities (Figure 2c):

$$
\bar{\varphi}=\frac{\sum_{i=1}^{n} I_{i} \varphi_{i}}{\sum_{i=1}^{n} I_{i}}
$$

where $\varphi_{i}$ and $I_{i}$ are the position and the intensity of maximum number $i$. In an ideal case, a peak contains a single maximum, and $\bar{\varphi}$ equals the $\varphi$ position of the peak maximum (Figure 3a). In non-ideal cases, $\bar{\varphi}$ represents the angle, to which the stream as a whole heads toward (Figure $3 b-d$ ).

Stream width. To characterize the stream width, we suggest an average full width at half maximum (FWHM) $\bar{\omega}$ of all sections, excluding transition sections:

$$
\bar{\omega}=\frac{1}{n} \sum_{i=1}^{n} \omega_{i}
$$

where $\omega_{i}$ is the FWHM of section number $i$ in the angulagram (see Figure 2c). In transition sections, the stream transits from one angle to another for further propagation. Transition sections possess no maxima and their intensity lines are parallel to the $\varphi$ axis in the angulagram.

Stream linearity. Based on the found values of $\bar{\varphi}$ and $\bar{\omega}$, a peak shape on the angulagram of a linear stream (e.g. black curves in Figure 2a and Figure 3) can be calculated by:

$$
P(\varphi):=P_{\bar{\varphi}, \bar{\omega}}(\varphi)=I_{\max } \frac{\bar{\omega}^{2}}{\bar{\omega}^{2}+(\bar{\varphi}-\varphi)^{2}}+I_{0}
$$

where $I_{\max }$ is the maximum intensity and $I_{0}$ is the background intensity. In an ideal case, $P(\varphi)$ is identical to the measured signal $S(\varphi)$ for the stream (Figure 3a), while in non-ideal cases, $P(\varphi)$ deviates from $S(\varphi)$ to various extents (Figure $3 b-d)$.

To characterize stream linearity, we suggest a measure of closeness of $S(\varphi)$ to $P(\varphi)$ expressed by R-squared (coefficient of determination) determined by least squares fitting. In order to not confuse $\mathrm{R}$-squared with resolution (denoted as $R$ ) we use $L^{2}$ as its symbol for the linearity parameter (Figure 2d):

$$
L^{2}=\left(\frac{\sum\left(p_{\mathrm{d}}-\bar{p}_{\mathrm{d}}\right)\left(s_{\mathrm{d}}-\bar{s}_{\mathrm{d}}\right)}{\sqrt{\sum\left(p_{\mathrm{d}}-\bar{p}_{\mathrm{d}}\right)^{2} \sum\left(s_{\mathrm{d}}-\bar{s}_{\mathrm{d}}\right)^{2}}}\right)^{2}
$$

where $p_{\mathrm{d}}$ and $s_{\mathrm{d}}$ are discrete data points for $P(\varphi)$ and $S(\varphi)$, respectively, and $\bar{p}_{\mathrm{d}}$ and $\bar{s}_{\mathrm{d}}$ are the respective averages of these data points. Note that $L^{2}$ is a measure of closeness (proximity) not distance, i.e. $L^{2}$ is a very sensitive measure and drops very rapidly with increasing non-linearity (Figure $3 a-d)$ ). If required by the application, measures for the distance between $S(\varphi)$ and $P(\varphi)$ are provided by Hausdorff or Fréchet metrics. ${ }^{15,16}$

Stream parameters as a guide for MSS improvement. The above definitions of quantitative parameters for stream deflection, stream width, and stream linearity allow the development of the third and last tool for the proposed toolbox: the utilization of these parameters for finding deficiencies and guiding improvements in MSS. Note that we focus here on steady-state MSS.

The theoretical stream deflection is represented by $\varphi_{\text {theo, }}$, which is a function of $v_{\text {flow }}$ and $v_{\text {disp }}$ :

$$
\tan \varphi_{\text {theo }}=\frac{v_{\text {disp }}}{v_{\text {flow }}}
$$

The value of $v_{\text {disp }}$ is directly proportional to the discriminative separation force (e.g. induced by an electric field in FFE) exerted upon molecules of an individual stream. The sign of $\varphi_{\text {theo }}$ depends on that of $v_{\text {disp }}\left(v_{\text {flow }}\right.$ is always positive). The sign 
of $v_{\text {disp }}$ is always positive in CAC, where all analytes are displaced in one direction. In FFE, the sign of $v_{\text {disp }}$ is the same as that of the electrophoretic mobility $\mu$ of the respective analyte (see SI 6). Another way to determine the sign of $v_{\text {disp }}$ in FFE is to compare directions of $\vec{v}_{\text {flow }}$ and the vector of electric field $\vec{E}: v_{\text {disp }}>0$ if $\vec{v}_{\text {flow }}$ and $\vec{E}$ are co-directed, and $v_{\text {disp }}<0$ if $\vec{v}_{\text {flow }}$ and $\vec{E}$ are counter-directed. Whether $\bar{\varphi}$ resembles $\varphi_{\text {theo }}$ depends on the presence of non-uniformities in $v_{\text {flow }}$ and $v_{\text {disp. }}$. In general, we consider a difference of more than $2^{\circ}$ between $\bar{\varphi}$ and $\varphi_{\text {theo }}$ as an indicator of unacceptably nonuniform velocity fields; this non-uniformity should accordingly be addressed, e.g. by redesigning or refabricating the device.

The stream width depends mainly on the overall velocity of the molecules and the velocity distribution along the height of the separation zone. The value of $\bar{\omega}$ should be low since broad streams require more effort to fully separate and collect them. From our experience and literature evaluation (SI 3), a stream should not occupy more than $15^{\circ}$ in $\bar{\omega}$ (Figure $3 \mathrm{a}, \mathrm{c}$ ); a very narrow and focused stream results in $\bar{\omega}$ of less than $10^{\circ}$ (Figure 3a). The issue of broader streams (Figure 3d) must be addressed by increasing the velocity of species (e.g. by increasing the $v_{\text {flow }}$ ) or by tuning certain properties of the separation media such as viscosity, $\mathrm{pH}$, and ionic strength.

The stream linearity represented by $L^{2}$ depends on the uniformity and stability of the velocity fields as well as the overall separation process. For a near-ideal stream (Figure 3a) the linearity is high $\left(L^{2} \geq 0.90\right)$, i.e. the stream is progressing without (much) disturbance through the separation zone. Peak splitting in various degrees (Figure $3 b-d$ ) results in $L^{2}<0.90$, which is an indicator for non-uniform velocity fields and instabilities in the system. Such non-uniform fields must be addressed, e.g. by redesigning or refabricating the device.

At first glance, it may seem redundant to characterize all
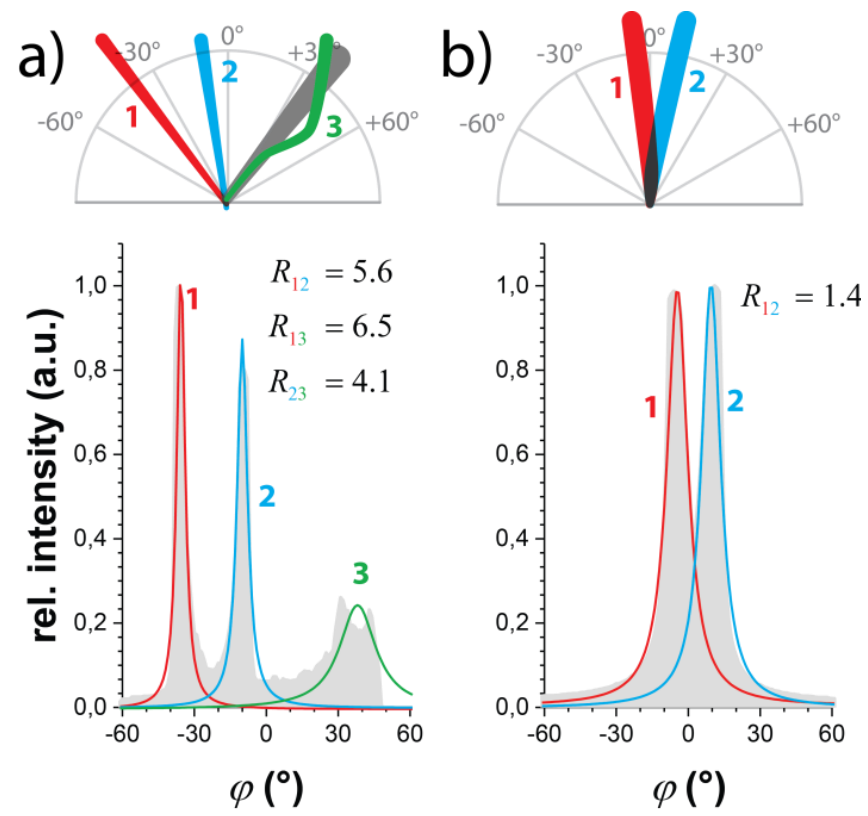

Figure 4. Stream resolution in cases of three well separated streams (a) and two worse separated streams (b). three parameters since they are all affected by non-uniform velocity fields. However, the type and magnitude of the influence of non-uniformity on the parameters is different. As we demonstrate below, a stream can appear linear and narrow but still be "misdeflected" indicating a non-uniform velocity field.

Based on the two parameters characterizing stream deflection and stream width, the common analytical measure of resolution $R$ can be calculated for two streams (Figure 4):

$$
R_{12}=\frac{2\left|\bar{\varphi}_{2}-\bar{\varphi}_{1}\right|}{\left(\bar{\omega}_{1}+\bar{\omega}_{2}\right)}
$$

where the subscripts designate peak numbers. $R$ should be greater than 1.0 for the streams to be collectable without overlap at $r_{\max }(\varphi)$. Low $R$ can be countered by decreasing $\bar{\omega}$ (see above) and/or increasing the difference in $\bar{\varphi}$. To increase the difference in $\bar{\varphi}$, the magnitude of the differential velocity vector $\Delta \vec{v}_{\text {disp }}=\vec{v}_{\text {disp , } 2}-\vec{v}_{\text {disp, } 1}$ must be increased. This increase can be achieved, to some extent, by increasing the discriminative force (e.g. that induced by the electric field in FFE). Alternatively, $v_{\text {flow }}$ can be decreased to increase the residence time of molecules in the MSS separation zone. The latter approach is limited, though, since decreasing $v_{\text {flow }}$ will eventually lead to an increase in $\bar{\omega}$. Note, that some species are only separable by switching the underlying separation method (e.g. CAC instead of FFE).

The three parameters characterizing stream deflection, stream width, and stream linearity, as well as stream resolution, can be observed over time to make further conclusions about the stability of the separation. Ideally, all quantities are stable and only vary slightly (we use $\leq 2^{\circ}, \leq 4^{\circ}$, $\leq 0.05$, and $\leq 0.5$ as acceptable deviations for $\bar{\varphi}, \bar{\omega}, L^{2}$, and $R$, respectively) over time. Strong time dependence of one or more parameters suggests instability, e.g. induced by a pulsing pressure profile of a pump responsible for controlling $v_{\text {flow }}$, which should be addressed to maintain steady-state MSS (e.g. by using a pulsation dampener).

Instructiveness of stream parameters. We stated in the beginning that the proposed analytical toolbox for quantitative characterization of stream quality is required for the advancement of MSS. To fulfil this requirement, application of such a toolbox must allow instructive conclusions. By the following examples of analyses of FFE experiments from the literature, we demonstrate that our toolbox can provide such instructions. We could not find any images of $\mathrm{CAC}$ separations for digitization and further characterization. Imaging the rotating separation zone in CAC is a challenge; due to the lack of image-analysis tools such as introduced here, there was no benefit to overcome this challenge, yet. In future, imaging systems in combination with our analytical tools will allow CAC developers to characterize and subsequently advance, for example, the packaging uniformity, which is a critical point in CAC. ${ }^{5}$

The raw FFE data of the following two experiments were taken from publications by Jezierski et al. and Kohlheyer et $a l . ;^{12,13}$ the angulagrams, constructed by us, can be found in Figure 5. The following is a summary of our analysis described in detail in SI 6 for Jezierski et al and in SI 7 for Kohlheyer et al.

Jezierski et al. performed MSS of four analytes, whose streams were assigned by the authors to fluorescein (Stream 1), sulforhodamine B (Stream 2), rhodamine B 
(Stream 3), and rhodamine 6G (barely visible Stream 4) (Figure 5a). The values for $\bar{\omega}$ and $L^{2}$ are $<5^{\circ}$ and $\geq 0.98$, respectively, suggesting very narrow and linear streams. For further analysis, we compared $\bar{\varphi}$ to $\varphi_{\text {theo }}$; the latter was calculated with $v_{\text {flow }}$ specified in the source paper, ${ }^{12}$ and $v_{\text {disp }}=\mu \times E$, where $\mu$ in the electrophoretic mobility found in the independent literature, ${ }^{17-19}$ and $E$ is electric field strength from the source paper. ${ }^{12}$ We found that for fluorescein (Stream 1) and sulforhodamine B (Stream 2), $\bar{\varphi}$ and $\varphi_{\text {theo }}$ are in agreement, while for rhodamines $\mathrm{B}$ and $6 \mathrm{G}$ they disagree; coincidently, $\bar{\varphi}$ for Stream 3 is equal to $\varphi_{\text {theo }}$ for rhodamine 6G. In summary, our analysis suggests that rhodamines B and $6 \mathrm{G}$ are co-migrating in Stream 3 , and that none of the 4 sample molecules can be assigned to Stream 4, which has the opposite $\bar{\varphi}$ to that of fluorescein.

Kohlheyer et al. performed MSS separation of a mixture of fluorescein (Stream 1) and rhodamine B (Stream 2) (Figure 5b). The values for $\bar{\omega}$ and $L^{2}$ are $<4^{\circ}$ and $\geq 0.96$, respectively, suggesting very narrow and linear streams. Analogously to the first example, we compared $\bar{\varphi}$ of both streams with the respective $\varphi_{\text {theo }}$ and found that $\bar{\varphi}$ does not match its respective $\varphi_{\text {theo }}$ for fluorescein, while $\varphi_{\text {theo }}$ and $\bar{\varphi}$ appear to be in agreement for rhodamine B. Our analysis suggests that $\vec{v}_{\text {flow }}$ is not uniform within the separation zone, and that there are flow streamlines moving with $v_{\text {flow }}$ as high as $22 \mathrm{~mm} \mathrm{~s}^{-1}$ in a 10-mm-long separation zone, while Kohlheyer et al. imply uniform $v_{\text {flow }}=3 \mathrm{~mm} \mathrm{~s}^{-1}$. Such MSSs are hard to model, and molecular stream behaviours in them are hard to predict. The full assessment of such MSSs requires measuring their velocity fields; we recently developed a comprehensive approach (including hardware, algorithms, and software) for such measurements. ${ }^{20,21}$

Most literature expectably presents only 'good' cases, i.e. narrow, linear streams with a good separation. However, evaluation by angulagrams is especially useful for 'bad' cases. Therefore, we complement the analysis of above 'good' literature data by a brief analysis of separation images from our own publication as a 'bad' examples (Figure 5c,d; SI 8). ${ }^{20,21}$ These separations were 'bad' by design to demonstrate the capabilities of our imaging system. We performed MSS separation of fluorescein (Stream 1) and rhodamine $6 \mathrm{G}$ (Stream 2 ) in both cases. The values for $\bar{\omega}$ and $L^{2}$ are $<15^{\circ}$ and $<0.40$, respectively, suggesting narrow but non-linear streams. The peak splitting and the shape of peaks (see SI 4 for details) present in the angulagrams show that all streams are bending in the separation zone. Unsurprisingly, we found than $\bar{\varphi}$ of all streams do not match with the respective $\varphi_{\text {theo }}$. Comparing the misshape of peaks in these angulagrams (Figure 5c,d) with the near-Lorentzian shapes in the cases above (Figure 5a,b) reveals that not only $v_{\text {flow }}$ is not uniform in our 'bad' examples, but the separation is highly disturbed and very likely not steady-state. The device may have become clogged or the manufacture process created an uneven separation zone; hence, the device needs to be replaced.

Comparison with classical cross-section approaches. The above examples demonstrate that finding and validating $\bar{\varphi}, \bar{\omega}$, and $L^{2}$ allows one: (i) to identify deficiencies in MSS for which raw data (images) appear fine and (ii) to draw instructive conclusions for reasons of these deficiencies and ways of their elimination. So far, in the absence of our tools, raw MSS data were evaluated by analysing signal in a single cross-section perpendicular to $\vec{v}_{\text {flow }}{ }^{5,11-14,22}$ A cross-section
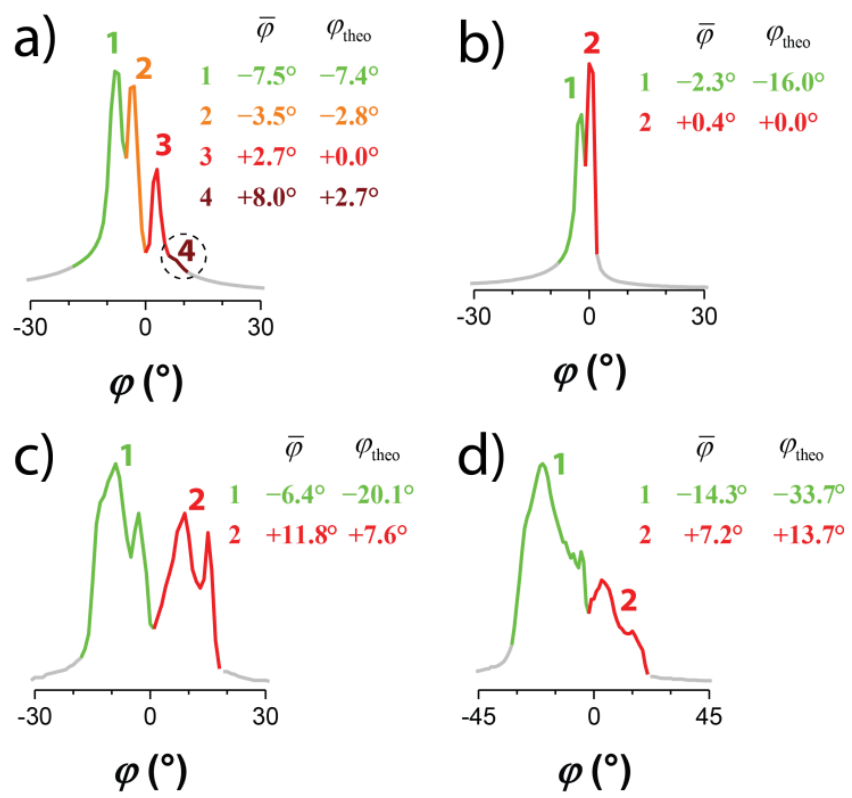

Figure 5. Comparing measured with expected stream deflections in angulagrams constructed with data published by Jezierski et al. $(\mathbf{a})^{12}$, Kohlheyer et al. (b) $)^{13}$, and ourselves $(\mathbf{c}, \mathbf{d}){ }^{20,21} \bar{\varphi}$ is the position of the maximum of the respective peak. Analytes were assigned to the streams by the authors of source data. $\varphi_{\text {theo }}$ was calculated with $v_{\text {flow }}$ and $v_{\text {disp }}\left(v_{\text {disp }}=\mu \times E\right)$ found in the literature. ${ }^{14-16}$ For more details (including replicates of original images) see SI 3, SI 6, SI 7, and SI 8.

reduces dimensionality of MSS data from 3D to 2D and allows the extraction of peak width and peak position, which are related to our $\bar{\omega}$ and $\bar{\varphi}$. However, a cross-section represents only a very small part of the separation zone, and, accordingly, parameters extracted from a cross-sectional signal are only valid at the position of cross-section. A cross-section possesses no record of the stream's spatial history, which is required to assess stream linearity $\left(L^{2}\right)$. In essence, a crosssection reduces the dimensionality by cutting out important information (in contrast to our approach in which 3D information is convoluted into a $2 \mathrm{D}$ angulagram). Finally, the cross-sectional approach makes it hard, if not impossible, to compare two different MSSs without their elaborate normalization (e.g. for scale). Even with the normalization, one would compare only a small part of one MSS to a small part of another MSS, but never the whole MSS separation zones.

Applicability of our approach. Our analysis method is applicable to any steady-state MSS. It is not applicable to nonsteady state methods such as isotachophoresis or isoelectric focusing. Furthermore, we discussed and developed our approach considering $\vec{v}_{\text {flow }}$ and $\vec{v}_{\text {disp }}$ being unidirectional, which is the most common case; it might not be directly applicable to the more complex cases with non-unidirectional velocity fields. However, future developments might derive similar concepts for these methods and, thus, increase the scope of application. 


\section{CONCLUSION}

To summarize, we introduce a toolbox consisting of three tools for quantitative characterization of MSS. Tool 1 is our method of convoluting 3D raw MSS data into a 2D angulagram. Angulagrams are easy to construct from simple images; this does not require any information about the scale or dimensions of the respective MSS separation zone, which allows the straightforward comparison of differently scaled MSSs without any elaborate normalization. Tool 2 is three scalar parameters characterizing stream deflection, steam width, and stream linearity. These parameters are straightforward to extract from an angulagram by dissecting peaks. Tool 3 is the analysis of these parameters and derivatives such as stream resolution in relation to physicochemical characteristics of MSS. This kind of analysis offers MSS developers and users indications for non-uniform velocity fields and non-ideal separations; such indications reveal deficiencies and guide improvements in MSS devices and methods. We foresee that our toolbox will stimulate the development of MSS as a scientific and technological field and aid MSS advancement.

\section{ASSOCIATED CONTENT}

\section{Supporting Information}

The Supporting Information is available free of charge on the ACS Publications website.

- Details on equations, angulagrams, and literature examples

- Programs to construct angulagrams from images

- Example data

\section{AUTHOR INFORMATION}

\section{Corresponding Author}

* Sergey N. Krylov. E-mail: skrylov@yorku.ca Homepage: http://www.yorku.ca/skrylov/

\section{ORCIDs}

Sven Kochmann: 0000-0001-7423-4609

Sergey N. Krylov: 0000-0003-3270-2130

\section{Author Contributions}

The manuscript was written through contributions of all authors. All authors have given approval to the final version of the manuscript.

\section{ACKNOWLEDGMENT}

This work was supported by a Strategic Projects Grant 463455 from NSERC Canada. The authors thank Dr. Victor Galievsky and Dr. Alexander Stasheuski for valuable discussions and suggestions.

\section{REFERENCES}

(1) Giddings, J. C. Transport, space, entropy, diffusion and flow: Elements underlying separation by electrophoresis, chromatography, field-flow fractionation and related methods. J. Chromatogr. A 1987, $395,19-32$.
(2) Giddings, J. C. Unified Separation Science; Wiley, 1991.

(3) Denoroy, L.; Zimmer, L.; Renaud, B.; Parrot, S. Ultra high performance liquid chromatography as a tool for the discovery and the analysis of biomarkers of diseases: A review. J. Chromatogr. B 2013, 927, 37-53.

(4) Ramos-Payan, M.; Ocana-Gonzalez, J. A.; Fernandez-Torres, R. M.; Llobera, A.; Angel Bello-Lopez, M. Recent trends in capillary electrophoresis for complex samples analysis: A review. Electrophoresis 2018, 39, 111-125.

(5) Hilbrig, F.; Freitag, R. Continuous annular chromatography. J. Chromatogr. B 2003, 790, 1-15.

(6) Johnson, A. C.; Bowser, M. T. Micro free flow electrophoresis. Lab Chip 2017, 18, 27-40.

(7) Novo, P.; Janasek, D. Current advances and challenges in microfluidic free-flow electrophoresis-A critical review. Anal. Chim. Acta 2017, 991, 9-29.

(8) Agostino, F. J.; Cherney, L. T.; Galievsky, V.; Krylov, S. N. Steady-State Continuous-Flow Purification by Electrophoresis. Angew. Chem. Int. Ed. 2013, 52, 7256-7260.

(9) Jezierski, S.; Tehsmer, V.; Nagl, S.; Belder, D. Integrating continuous microflow reactions with subsequent micropreparative separations on a single microfluidic chip. Chem. Commun. 2013, 49, 11644-11646.

(10) Pfeiffer, S. A.; Rudisch, B. M.; Glaeser, P.; Spanka, M.; Nitschke, F.; Robitzki, A. A.; Schneider, C.; Nagl, S.; Belder, D. Continuous purification of reaction products by micro free-flow electrophoresis enabled by large area deep-UV fluorescence imaging. Anal. Bioanal. Chem. 2018, 410, 853-862.

(11) Cheng, L.-J.; Chang, H.-C. Switchable pH actuators and 3D integrated salt bridges as new strategies for reconfigurable microfluidic free-flow electrophoretic separation. Lab on a Chip 2014, 14, 979-987.

(12) Jezierski, S.; Gitlin, L.; Nagl, S.; Belder, D. Multistep liquidphase lithography for fast prototyping of microfluidic free-flowelectrophoresis chips. Anal. Bioanal. Chem. 2011, 401, 2651-2656.

(13) Kohlheyer, D.; Besselink, G. A. J.; Schlautmann, S.; Schasfoort, R. B. M. Free-flow zone electrophoresis and isoelectric focusing using a microfabricated glass device with ion permeable membranes. Lab Chip 2006, 6, 374-380.

(14) Raymond, D. E.; Manz, A.; Widmer, H. M. Continuous Sample Pretreatment Using a Free-Flow Electrophoresis Device Integrated onto a Silicon Chip. Anal. Chem. 1994, 66, 2858-2865.

(15) Efrat; Guibas; Har-Peled, S.; Mitchell; Murali. New Similarity Measures between Polylines with Applications to Morphing and Polygon Sweeping. Discrete Comput. Geom. 2002, 28, 535-569.

(16) Fréchet, M. M. Sur quelques points du calcul fonctionnel. Rend. Circ. Mat. Palermo (1884-1940) 1906, 22, 1-72.

(17) Breadmore, M. C.; Quirino, J. P.; Thormann, W. Insight into the mechanism of transient trapping in micellar electrokinetic chromatography. Electrophoresis 2011, 32, 542-549.

(18) Milanova, D.; Chambers, R. D.; Bahga, S. S.; Santiago, J. G. Electrophoretic mobility measurements of fluorescent dyes using onchip capillary electrophoresis. Electrophoresis 2011, 32, 3286-3294.

(19) Milanova, D.; Chambers, R. D.; Bahga, S. S.; Santiago, J. G. Effect of PVP on the electroosmotic mobility of wet-etched glass microchannels. Electrophoresis 2012, 33, 3259-3262.

(20) Kochmann, S. 2017, Schallaven/ffeimg: Initial release under GPL, DOI: 10.5281/zenodo.1001785.

(21) Kochmann, S.; Krylov, S. N. Image processing and analysis system for development and use of free flow electrophoresis chips. Lab Chip 2017, 17, 256-266.

(22) Castro, E. R.; Manz, A. Present state of microchip electrophoresis: State of the art and routine applications. $J$. Chromatogr. A 2015, 1382, 66-85. 